\title{
A HOMOTOPY THEOREM FOR MATROIDS, I
}

BY

W. T. TUTTE

1. Introduction. By a matroid on a finite set $M$ we understand a class $M$ of non-null subsets of $M$ which satisfies the following axioms.

Axiom I. No member of $\boldsymbol{M}$ contains another as a proper subset.

Axiom II. If $(X, Y) \in M, a \in X \cap Y$ and $b \in X-(X \cap Y)$, then there cxists $Z \in M$ such that $b \in Z \subseteq(X \cup Y)-\{a\}$.

Such systems were introduced by Hassler Whitney [1].

As an example let $L$ be any class of subsets of $M$ forming a group under mod 2 addition, and let $M$ be the class of all minimal non-null members of $L$. Then it is easily verified that $L$ satisfies Axiom II and that each non-null member of $L$ is a sum of non-null members of $M$. It follows that $M$ satisfies both axioms and is thus a matroid. Such a matroid we call binary.

In particular $M$ may be the set of edges of a finite graph $G$ and $L$ may be the class of 1-cycles mod 2 of $G$. Then it is found that the members of $\boldsymbol{M}$ are those sets of edges of $G$ which define circuits. In this case we call $M$ the circuit-matroid of $G$.

Given a matroid $\boldsymbol{M}$ let $Q$ be the class of all unions of members of $\boldsymbol{M}$. Then each element of $Q$ is a subset of $M$. We partition $Q$ into disjoint classes $Q_{-1}, Q_{0}, Q_{1}, Q_{2}, \cdots$ according to the following rules.

(i) The null subset $\varnothing$ of $M$, considered as an cmpty union, is the only member of $Q_{-1}$.

(ii) When $Q_{r}$ has been determined for $-1 \leqq r \leqq k$ we define $Q_{k+1}$ as the class of all minimal members of

$$
P_{k}=Q-\bigcup_{r=-1}^{k} Q_{r}
$$

That is $\boldsymbol{Q}_{k+1}$ consists of all members of $\boldsymbol{P}_{k}$ which have no other members of $\boldsymbol{P}_{k}$ as a subset. The members of $\boldsymbol{Q}$ are the flats of $\boldsymbol{M}$. Those belonging to $\boldsymbol{Q}_{d}$ are the flats of dimension $d$, or $d$-flats.

At the end of $\S 2$ of this paper we interpret the dimensions of the flats of a circuit-matroid in terms of graph theory.

We shall see that the flats of a matroid $M$ on a set $M$ have some properties resembling those of the elements of a projective geometry. Because of this analogy we refer to the 0 -flats, 1 -flats and 2 -flats of $\boldsymbol{M}$ as its points, lines and pianes respectively. The points are simply the members of the class $M$.

We have to recognize one distinction which has no analogue in projective

Received by the editors October 26, 1956. 
geometry. A flat $F$ is disconnected if it can be represented as the union of two disjoint non-null subsets $F^{\prime}$ and $F^{\prime \prime}$ of $M$ such that each point $X$ of $\boldsymbol{M}$ satisfying $X \subseteq F$ satisfies also either $X \subseteq F^{\prime}$ or $X \subseteq F^{\prime \prime}$. If no such representation is possible then $F$ is connected. Thus the points of $\boldsymbol{M}$ and its (-1)-flat are connected.

A path in $M$ is a finite sequence $P=\left(X_{1}, \cdots, X_{k}\right)$ of one or more points of $M$, not necessarily all distinct, such that any two consecutive terms are distinct points of $\boldsymbol{M}$ which are subsets of the same connected line. The first and last terms of $P$ are its origin and terminus respectively. If they are the same point we call $P$ re-entrant. If $P$ has only one term we call it degenerate.

If $P=\left(X_{1}, \cdots, X_{k}\right)$ and $P^{\prime}=\left(X_{k}, \cdots, X_{m}\right)$ are paths of $\boldsymbol{M}$ such that the origin of $P^{\prime}$ is the terminus of $P$ then we define their product $P P^{\prime}$ as the path $\left(X_{1}, \cdots, X_{k}, \cdots, X_{m}\right)$. Multiplication of paths is clearly associative. It is therefore permissible to write a path $(P Q) R$ or $P(Q R)$ simply as $P Q R$.

Suppose we have two paths $P R$ and $P Q R$ where $Q$ is either (i) of the form $(X, Y, X)$ or (ii) of the form $(X, Y, Z, X)$ with $X, Y$ and $Z$ subsets of the same plane. Then we say that each of $P R$ and $P Q R$ can be derived from the other by an elementary deformation. Two paths $P_{1}$ and $P_{2}$ are homotopic if they are identical or if one can be derived from the other by a finite sequence of elementary deformations. Homotopy is clearly an equivalence relation. A path homotopic to a degenerate path is said to be null-homotopic.

In this paper we show that every re-entrant path in a matroid is nullhomotopic. Actually we prove a more general theorem, as the result just stated is not sufficient for the purposes of Paper II. We first agree to call a subclass $\boldsymbol{C}$ of $\boldsymbol{M}$ convex if it has the following property: if two distinct members $X$ and $Y$ of $C$ are subsets of the same line $L$ then every point of $\boldsymbol{M}$ which is a subset of $L$ is a member of $\boldsymbol{C}$. Given a convex subclass $\boldsymbol{C}$ of $\boldsymbol{M}$ we say that a path $P$ is off $\boldsymbol{C}$ if no term of $P$ is a point of $C$. We then enquire into the condition that a path $P$ off $\boldsymbol{C}$ can be transformed into a degenerate path by a finite sequence of elementary deformations so that all the intermediate paths are off $\boldsymbol{C}$. In this paper we show how the idea of an elementary deformation must be generalized so as to make this transformation possible for every re-entrant path $P$ off $\boldsymbol{C}$.

It is hoped that the technique here developed for the study of matroids will be found useful in graph theory when applied to the circuit-matroids of graphs.

2. Flats. Let $M$ be a matroid on a set $M$. We refer to the elements of $M$ as the cells of the matroid. If $S$ and $T$ are subsets of $M$ we use the symbol $S \subset T$ to denote that $S$ is a proper subset of $T$. We write $\langle S\rangle$ for the union of all the points of $M$ which are subsets of $S$. If $S$ is a flat of $M$ we denote its dimension by $d S$.

(2.1) If $S$ is a flat of $M$ and $k$ is an integer satisfying $-1 \leqq k<d S$ then there exists a flat $T$ of $M$ such that $d T=k$ and $T \subset S$.

Proof. If the theorem fails let $k$ be the greatest integer satisfying -1 
$\leqq k<d S$ such that no $k$-flat $T$ of $M$ satisfies $T \subset S$. Clearly $k>-1$ and therefore $d S \geqq 1$.

By the definition of $k$ there exists a $(k+1)$-flat $T^{\prime}$ of $M$ such that $T^{\prime} \subseteq S$. By the definition of the classes $Q_{r}$ we have

$$
T^{\prime} \in Q-\bigcup_{r=-1}^{k} Q_{r} \subset Q-\bigcup_{r=-1}^{k-1} Q_{r}=P_{k-1}
$$

But $T^{\prime}$ is not a minimal member of $P_{k-1}$, since $d T^{\prime}$ is not $k$. Hence there exists a minimal member $T$ of $P_{k-1}$ such that $T \subset T^{\prime}$. But then $d T=k$. Since $T^{\prime} \subseteq S$ this contradicts the definition of $k$. The theorem follows.

(2.2) If $S$ and $T$ are flats of $M$ such that $S \subset T$, then $d S<d T$.

Proof. Since $T$ is non-null we have $d T>-1$. If $d S \geqq d T$ there is a flat $U$ of $M$ such that $U \subseteq S$ and $d U=d T$, by (2.1). But then $T$ is not a minimal member of $\boldsymbol{Q}_{d T}$, contrary to the definition of this class.

It follows from (2.2) that $\langle M\rangle$ has a greater dimension than any other flat of $\boldsymbol{M}$.

It is convenient to say that a flat $S$ is on a flat $T$ if either $S \subseteq T$ or $T \subseteq S$. If $S$ and $T$ are distinct we can distinguish between the two cases by comparing dimensions.

(2.3) If $S$ is a flat of $M$ and $a \in S$, then $d\langle S-\{a\}\rangle=d S-1$.

Proof. If possible choose $S$ and $a$ so that $d\langle S-\{a\}\rangle \neq d S-1$ and so that $d S$ has the least value consistent with this.

By (2.1) there is a flat $T$ of $\boldsymbol{M}$ such that $T \subseteq S$ and $d T=d S-1$. Choose $b \in S-T$. Then $T \subseteq\langle S-\{b\}\rangle \subset S$. Hence $d\langle S-\{b\}\rangle=d S-1$, by (2.2).

Suppose $a \notin\langle S-\{b\}\rangle$. Then $\langle S-\{b\}\rangle \subseteq\langle S-\{a\}\rangle \subset S$. Hence $d\langle S-\{a\}\rangle$ $=d S-1$, by (2.2). But this is contrary to the choice of $S$ and $a$.

We deduce that $a \in\langle S-\{b\}\rangle$. Hence there exists $X \in M$ such that $X \subseteq S$, $a \in X$ and $b \in X$. Since $b \in S$ there exists $Y \in M$ such that $Y \subseteq S$ and $b \in Y$. It follows by Axiom II that there exists $Z \in M$ such that $Z \subseteq S, a \in Z$ and $b \in Z$. $(Z=Y$ if $a \in Y)$. These results imply

$$
\begin{aligned}
& \langle\langle S-\{a\}\rangle-\{b\}\rangle \subset\langle S-\{a\}\rangle \subset S, \\
& \langle\langle S-\{b\}\rangle-\{a\}\rangle \subset\langle S-\{b\}\rangle \subset S .
\end{aligned}
$$

We also have

$$
\langle\langle S-\{a\}\rangle-\{b\}\rangle=\langle\langle S-\{b\}\rangle-\{a\}\rangle,
$$

since each side of this equation represents the union of those points of $\boldsymbol{M}$ which include neither $a$ nor $b$.

Since $d\langle S-\{b\}\rangle=d S-1$ it follows from (2.3b) and the choice of $S$ and $a$ that $d\langle\langle S-\{b\}\rangle-\{a\}\rangle=d S-2$. Hence $d\langle S-\{a\}\rangle=d S-1$, by (2.2), (2.3a) and $(2.3 \mathrm{c})$. This contradiction establishes the theorem.

(2.4) Let $S$ and $T$ be flats of $\boldsymbol{M}$ such that $S \subseteq T$. Then there exists a flat $U$ of $\boldsymbol{M}$ such that $U \subseteq T,\langle U \cap S\rangle=\varnothing$ and $d U=d T-d S-1$. 
Proof. Write $S_{0}=S, T_{0}=T$. If possible choose $a_{0} \in S_{0}$ and write $S_{1}$ $=\left\langle S_{0}-\left\{a_{0}\right\}\right\rangle, T_{1}=\left\langle T_{0}-\left\{a_{0}\right\}\right\rangle$. Observe that $S_{1} \subseteq T_{1}$. If possible choose $a_{1} \in S_{1}$ and write $S_{2}=\left\langle S_{1}-\left\{a_{1}\right\}\right\rangle, T_{2}=\left\langle T_{1}-\left\{a_{1}\right\}\right\rangle$. Then $S_{2} \subseteq T_{2}$. Continue this process until it terminates. By (2.3) this will be with $S_{k}$ and $T_{k}$, where $k=d S+1$ and $S_{k}=\varnothing$. Applying (2.3) to the sequence of the $T_{i}$ we find that $d T_{k}=d T-k=d T-d S-1$. We note that $\left\langle T_{k} \cap S\right\rangle \subseteq\left\langle S-\left\{a_{0}, \cdots, a_{k-1}\right\}\right\rangle$ $=S_{k}=\varnothing$. Hence the theorem is satisfied with $U=T_{k}$.

(2.5) If $S$ and $T$ are any flats of $M$ then $d(S \cup T)+d\langle S \cap T\rangle \geqq d S+d T$.

Proof. Write $S_{0}=S$. If possible choose $a_{0} \in S_{0}-\left(S_{0} \cap T\right)$ and write $S_{1}$ $=\left\langle S_{0}-\left\{a_{0}\right\}\right\rangle$. If possible choose $a_{1} \in S_{1}-\left(S_{1} \cap T\right)$ and write $S_{2}=\left\langle S_{1}-\left\{a_{1}\right\}\right\rangle$, and so on. By (2.3) the process terminates with $S_{k}=\langle S \cap T\rangle$, where $k=d S$ $-d\langle S \cap T\rangle$. We now have $T=S_{k} \cup T \subset S_{k-1} \cup T \subset \cdots \subset S_{0} \cup T=S \cup T$. Hence $d(S \cup T)-d T \geqq k=d S-d\langle S \cap T\rangle$, by (2.2).

Many "geometrical" results can be deduced from (2.2) and (2.5). For example any two distinct lines $L_{1}$ and $L_{2}$ on a plane $P$ have a unique common point. To prove this we first use (2.2) to show that $d\left\langle L_{1} \cap L_{2}\right\rangle\left\langle d L_{1}=1\right.$ and $L_{1} \cup L_{2}=P$. Then $d\left\langle L_{1} \cap L_{2}\right\rangle \geqq 0$, by (2.5). Hence $d\left\langle L_{1} \cap L_{2}\right\rangle=0$ and $\left\langle L_{1} \cap L_{2}\right\rangle$ is a single point of $M$. We can prove in the same way that if $P_{1}$ and $P_{2}$ are distinct planes on the same 3-flat $E$ of $\boldsymbol{M}$, then $\left\langle P_{1} \cap P_{2}\right\rangle$ is a line on $E$. Similarly if $P$ is a plane and $L$ a line on the same 3-flat $E$, and $L$ is not on $P$, then $\langle P \cap L\rangle$ is a point on $E$.

Not all the axioms of projective geometry are valid for matroids. For example two points are not necessarily on a common line. In general matroids are like geometrical figures but not like complete geometries.

Suppose $M$ is the circuit-matroid of a graph $G$. If $S \in Q$ we write $G \cdot S$ for the subgraph of $G$ made up of the edges of $S$ and their incident vertices. We see that the flats $S$ of $M$ correspond to those subgraphs $G \cdot S$ in which each edge belongs to some circuit of the subgraph. In virtue of (2.3) $d S+1$ is the least number of edges which must be removed from $G \cdot S$ in order to destroy all its circuits, that is $d S+1$ is the rank or first Betti number of $G \cdot S$. The subgraph $G \cdot S$ is nonseparable if and only if the flat $S$ is connected.

3. Connected flats. We begin this section with a study of the line.

(3.1) Any line $L$ of $M$ is on at least two points. If $X$ and $Y$ are distinct points on $L$ then $L=X \cup Y$. Moreover $X \cap Y$ is non-null if and only if $L$ is connected.

Proof. Choose $a \in L$. Then $\langle L-\{a\}\rangle$ is a point on $L$, by (2.3). Choose $b \in\langle L-\{a\}\rangle$. Then $\langle L-\{b\}\rangle$ is a point on $L$, by (2.3), which is distinct from $\langle L-\{a\}\rangle$.

Let $X$ and $Y$ be distinct points on $L$. Then $X \subset X \cup Y \subseteq L$. Hence $X \cup Y$ $=L$, by (2.2). If $X \cap Y$ is non-null then $L$ is clearly connected. If $X \cap Y$ is null then either $L$ is disconnected or there exists $Z \in M$ such that $Z \subseteq X \cup Y$ and $Z$ meets both $X$ and $Y$. In the latter case $X \subset X \cup Z \subset X \cup Y=L$, by Axiom I. This is impossible, by (2.2). 
(3.2) A disconnected line is on just two points, and a connected line is on at least three points.

Proof. By (3.1) any two distinct points on a disconnected line $L$ are disjoint and have $L$ as their union. Hence $L$ has at most two points, and therefore just two by (3.1).

By (3.1) any connected line $L$ has two distinct points $X$ and $Y$, and we can find $a \in X \cap Y$. By (2.3) $\langle L-\{a\}\rangle$ is a point on $L$ distinct from $X$ and $Y$.

We shall need the following general theorems on connected flats.

(3.3) Let $S$ and $T$ be connected flats of $M$ such that $S \subset T$. Then there exists a connected $(d S+1)$-flat $U$ of $M$ which is on both $S$ and $T$.

Proof. Since $T$ is connected we can find $X \in M$ such that $X \subseteq T$ and $X$ meets both $S$ and $T-S$. Choose such an $X$ so that $S \cup X$ has the least possible number of cells. Clearly $S \cup X$ is a connected flat of $M$. Its dimension exceeds $d S$, by (2.2).

Suppose $d(S \cup X)>d S+1$. Choose $a \in(S \cup X)-S$. Then $d\langle(S \cup X)-\{a\}\rangle$ $\geqq d S+1$, by (2.3). Hence there exists $Y \in M$ such that $Y \subseteq(S \cup X)-\{a\}$ and $Y$ meets $(S \cup X)-S$. But $Y \cap S$ is null, by the choice of $X$. Hence $Y \subset X$, which is impossible by Axiom I. We deduce that $d(S \cup X)=d S+1$. Hence the theorem is true with $U=S \cup X$.

(3.4) Let $S$ be a connected $d$-flat on a connected $(d+2)$-flat $T$ of $M$. Then there exist distinct connected $(d+1)$-flats $U$ and $V$ of $M$ such that $S=\langle U \cap V\rangle$ and $T=U \cup V$.

Proof. By (3.3) there is a connected $(d+1)$-flat $U$ which is on both $S$ and $T$. Choose $a \in U-S$ and write $W=\langle T-\{a\}\rangle$. By (2.3) $W$ is another $(d+1)$-flat on $S$ and $T$. By (2.4) there is a line $L$ on $T$ having no point in common with $S$. It meets $U$ and $W$ in points $X$ and $Z$ respectively, by (2.5). (See Figure I.) By (2.2) we have $S \cup X=U$ and $S \cup Z=W$. Hence $Z$ is not on $U$ and therefore $U \cup Z=T$, by (2.2).

Assume $W$ is not connected. Then $S \cap Z=\varnothing$.

Suppose $U \cap Z=\varnothing$. By the connection of $T$ there exists $Z^{\prime} \in M$ such that $Z^{\prime} \subseteq T$ and $Z^{\prime}$ meets both $U$ and $Z$. Then $U \subset U \cup Z^{\prime} \subset U \cup Z=T$, by Axiom I. This is impossible by (2.2). We deduce that $U \cap Z \neq \varnothing$. A similar argument in which $X, S$ and $U$ replace $Z, U$ and $T$ respectively shows that $X \cap S \neq \varnothing$. Choose $b \in Z \cap U$ and $c \in X \cap S$.

Write $V=\langle T-\{b\}\rangle$. By (2.3) $V$ is a $(d+1)$-flat. It is on $S$ since $b \in Z$ and $S \cap Z=\varnothing$. By (2.5) it has a common point $Y$ with $L$. Clearly $V$ is distinct from $U$ and $W$ and therefore $Y$ is distinct from $X$ and $Z$, since $V=S \cup Y$ by (2.2).

Now $c \in S \cap X \subseteq S \cap L=S \cap(Y \cup Z)=S \cap Y$, by (3.1). Hence $V$ is connected.

If instead $W$ is connected we write $V=W$.

We now have two connected $(d+1)$-flats $U$ and $V$ of $M$ each of which is on both $S$ and $T$. Hence $S \subseteq\langle U \cap V\rangle \subset U \subset U \cup V \subseteq T$, since $U$ and $V$ are 


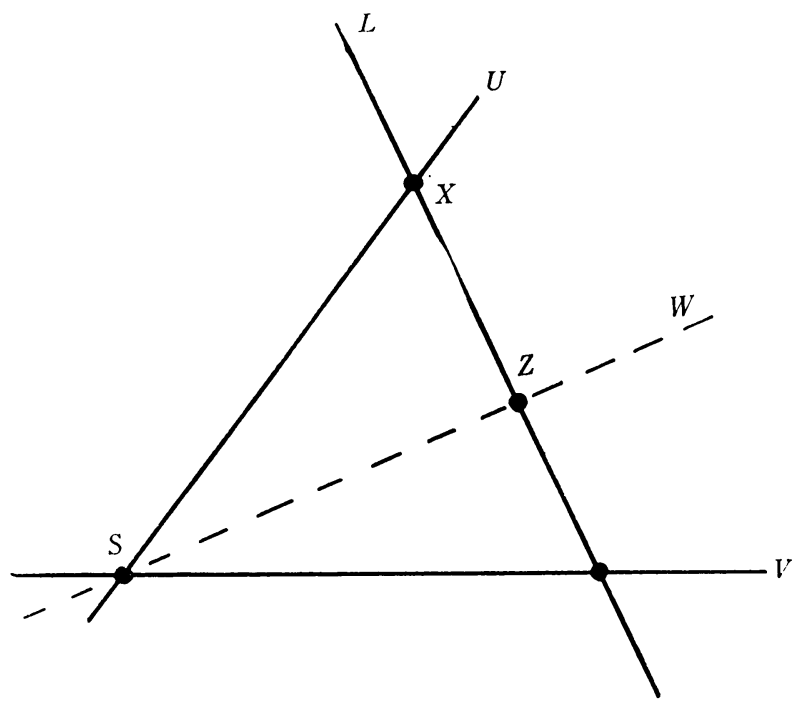

FIG. I

distinct and, by (2.2), neither is a subset of the other. In view of (2.2) this is possible only if $S=\langle U \cap V\rangle$ and $T=U \cup V$.

(3.5) Let $S, T$ and $U$ be flats of $M$ such that $S$ and $T$ are connected, $S \cup U \subseteq T$ and $\langle S \cap U\rangle=\varnothing$. Then there exists a connected flat $R$ of $M$ such that $S \subseteq R \subseteq T$, $\langle R \cap U\rangle=\varnothing$ and $d R=d T-d U-1$.

Proof. If possible choose $S, T$ and $U$ so that the theorem fails and $d U$ has the least value consistent with this. Then $d U>-1$ since otherwise the theorem holds with $T=R$. Let $W$ be a connected flat of $M$ of greatest possible dimension such that $S \subseteq W \subseteq T$ and $W$ does not contain $U$. Then $d W$ $=d T-1$ since otherwise, by (3.3) and (3.4), there exist distinct connected $(d W+1)$-flats $K$ and $L$ of $\boldsymbol{M}$ on $T$ such that $\langle K \cap L\rangle=W$, and these cannot both contain $U$. By the choice of $S, T$ and $U$ there is a connected flat $R$ of $M$ such that $S \subseteq R \subseteq W \subset T,\langle R \cap U\rangle=\varnothing$ and $d R=d W-d\langle U \cap W\rangle-1$. But then $d R \geqq d T-d U-1$, by (2.2), and therefore $d R=d T-d U-1$, by (2.2) and (2.5). This contradiction establishes the theorem.

The foregoing results can be applied to circuit-matroids to obtain rather simple theorems about graphs. Thus from (3.5) with $S=\varnothing$ we find that if a nonseparable graph $G$ has rank $r$ and a subgraph $G \cdot U$ has a rank $s$ then there is a nonseparable subgraph $G \cdot R$ of $G$ of rank $r-s$ having no circuit in common with $G \cdot U$.

4. The disconnected line. By a separation $\left\{S_{1}, S_{2}\right\}$ of a disconnected flat $S$ of $M$ we mean a pair of complementary non-null subsets of $S$ such that each $X \in M$ satisfying $X \subseteq S$ satisfies either $X \subseteq S_{1}$ or $X \subseteq S_{2}$.

(4.1) If $\left\{S_{1}, S_{2}\right\}$ is a separation of a flat $S$ of $M$ and $X_{1}$ and $X_{2}$ are 
points of $M$ such that $X_{1} \subseteq S_{1}$ and $X_{2} \subseteq S_{2}$, then $X_{1} \cup X_{2}$ is a disconnected line of $M$.

Proof. Suppose $Y$ is a point on $X_{1} \cup X_{2}$ distinct from $X_{1}$ and $X_{2}$. Since $Y \subseteq S$ we have $Y \subseteq S_{1}$ or $Y \subseteq S_{2}$. Hence $Y \subset X_{1}$ or $Y \subset X_{2}$, contrary to Axiom I. Thus the only subsets of $X_{1} \cup X_{2}$ which are flats of $M$ are $\varnothing, X_{1}, X_{2}$ and $X_{1} \cup X_{2}$. Hence, by the definition of dimension, $X_{1} \cup X_{2}$ is a line of $M$ having $\left\{X_{1}, X_{2}\right\}$ as a separation.

(4.2) Let $L$ be a disconnected line on a connected d-flat $S$ of $M$, where $d S>1$. Then there exists a connected plane $P$ of $M$ such that $L \subset P \subseteq S$.

Proof. Let the two points on $L$ be $X$ and $Y$. Let $P$ be a connected flat of $M$ of least possible dimension such that $L \subset P \subseteq S$. Assume $d P>2$.

Suppose first that there is a disconnected line $L^{\prime}$, distinct from $L$, on $X$ and $P$. Let its point other than $X$ be $Z$. By (3.5) there is a connected $(d P-2)$ flat $U$ on $Y$ and $P$ having no point in common with $L^{\prime}$. By (3.4) there are distinct connected $(d P-1)$-flats $V$ and $W$ of $M$ on $P$ such that $\langle V \cap W\rangle=U$. By (2.2) and (2.5) $V$ and $W$ meet $L^{\prime}$ in distinct points. Since there are only two points on $L^{\prime}$ we may suppose $X$ is on $V$. But then $L$ is on $V$ and the definition of $P$ is contradicted. A similar argument applies if there is a disconnected line distinct from $L$ on $Y$ and $P$.

In the remaining case we choose $a \in P-L$ and write $R=\langle P-\{a\}\rangle$. Then $L \subseteq R$. Moreover $d R=d P-1$, by (2.3). By the definition of $P$ the flat $R$ is disconnected. But there is no disconnected line on $R$, other than $L$, which is on either $X$ or $Y$. Hence, by (4.1), the only possible separation of $R$ is $\{X, Y\}$. Accordingly $R=L$ and $d P=2$, contrary to assumption. From this contradiction we deduce that $P$ is a plane.

(4.3) Let $L$ be a disconnected line on a connected plane $P$ of $M$. Let $X$ and $Y$ be the two points of $L$ and let $Z$ be any other point on $P$. Then $X \cup Z$ and $Y \cup Z$ are connected lines. Moreover they are the only lines of $M$ which are on both $Z$ and $P$.

Proof. Any line on $Z$ and $P$ has a common point with the line $X \cup Y$. Hence, by (3.1), the only flats on $Z$ and $P$ which can be lines are $X \cup Z$ and $Y \cup Z$. By (3.4) both these flats must be connected lines.

(4.4) Let $L$ be a disconnected line on a connected plane $P$ of $M$. Then every line on $P$ other than $L$ is connected.

Proof. Let $L^{\prime}$ be any such line. By (3.1) it is on a point $Z$ distinct from $X$ and $Y$. Hence, by (4.3) it is one of the connected lines $X \cup Z$ and $Y \cup Z$.

5. Convex subclasses. Convex subclasses of a matroid $M$ were defined in the Introduction. As an example we may take the class of all points of $\boldsymbol{M}$ on a given flat. The convexity of this class follows from (3.1).

Consider any path $P=\left(X_{1}, X_{2}, \cdots, X_{k}\right)$ of $\boldsymbol{M}$. We say $P$ is a path from $X_{1}$ to $X_{k}$. Any two consecutive terms of $P$ have a non-null intersection, by (3.1). Hence the flat $X_{1} \cup X_{2} \cup \ldots \cup X_{k}$ is connected. We denote this flat by $F(P)$. If $S$ is any flat of $M$ such that $F(P) \subseteq S$ we say that $P$ is a path $o n S$.

(5.1) Let $\mathbf{C}$ be any convex subclass of $M$. Let $S$ be a non-null connected flat 
of $M$ and let $X$ and $Y$ be points on $S$ such that $Y \notin C$. Then there exists a path $P$ from $X$ to $Y$ on $S$ such that no term of $P$ other than the first is a point of $C$.

Proof. If possible choose $S, X$ and $Y$ so that the theorem fails and $d S$ has the least value consistent with this. Clearly $d S>1$. By (3.3) and (3.4) there is a connected $(d S-2)$-flat $U$ and two distinct connected $(d S-1)$-flats $V$ and $W$ on $S$ such that $X \subseteq U=\langle V \cap W\rangle$. Now $Y$ is not on $V$ or $W$, for otherwise there would be a path from $X$ to $Y$ on $V$ or $W$ of the kind required. By (3.5) there is a connected line $L$ on $S$ and $Y$ such that $\langle L \cap U\rangle=\varnothing$. This meets $V$ and $W$ in distinct points $Z(V)$ and $Z(W)$ respectively, by (2.2) and (2.5). At least one of these, say $Z(V)$, belongs to $\boldsymbol{M}-\boldsymbol{C}$ since $\boldsymbol{C}$ is convex. By the choice of $S, X$ and $Y$ there is a path $Q$ from $X$ to $Z(V)$ on $V$ such that no term of $Q$ other than the first is a point of $C$. Adjoining $Y$ to $Q$ we obtain a path $P$ from $X$ to $Y$ on $S$ of the kind required. This contradiction establishes the theorem.

We now distinguish four kinds of re-entrant paths of $\boldsymbol{M}$ as elementary with respect to a given convex subclass $\boldsymbol{C}$ of $\boldsymbol{M}$. The first kind consists of all paths off $\boldsymbol{C}$ of the form $(X, Y, X)$. The second consists of all paths off $\mathbf{C}$ of the form $(X, Y, Z, X)$ such that $d(X \cup Y \cup Z) \leqq 2$.

Suppose $P$ is a plane of $M$ on which there are two distinct points $A$ and $B$ of $C$ such that each connected line on $P$ is on either $A$ or $B$. Then any path off $C$ on $P$ of the form $(X, Y, Z, T, X)$ such that $X, Y, Z$ and $T$ are distinct, the lines $X \cup Y$ and $Z \cup T$ are on $A$, and the lines $Y \cup Z$ and $T \cup X$ are on $B$ is an elementary re-entrant path of the third kind with respect to $C$.

Suppose $E$ is a 3 -flat of $M$ on which there are three points $A, B$ and $C$ such that $A \cup B, B \cup C$ and $C \cup A$ are disconnected lines. Let there be just six connected planes on $E$, two on each of these disconnected lines. Suppose $A, B$ and $C$ are all in $\boldsymbol{M}-\boldsymbol{C}$ but there are two distinct members of $\boldsymbol{C}$ on each of the six connected planes. Then any path off $C$ of the form $(A, X, B$, $Y, A)$, where $X$ and $Y$ are on distinct connected planes on $A \cup B$ and $E$, is an elementary re-entrant path of the fourth kind with respect to $C$.

In studying the preceding case it is convenient to use the following notation. We write $Z_{1}, Z_{2}$ and $Z_{3}$ for $A, B$ and $C$. We enumerate the six connected planes as $P_{1}, \cdots, P_{6}$ in such a way that $\left\langle P_{i} \cap P_{i+3}\right\rangle=Z_{j} \cup Z_{k}$, where $1 \leqq i \leqq 3$ and $(i, j, k)$ is a permutation of $(1,2,3)$. In general we write $\left\langle P_{i} \cap P_{j}\right\rangle=L_{i j}$ for $1 \leqq i<j \leqq 6$. If $j=i+3$ then $L_{i j}$ is the disconnected line $\left(Z_{1} \cup Z_{2} \cup Z_{3}\right)-Z_{i}$. If $j \neq i+3$ let $k$ be that integer 1,2 or 3 which is not congruent to $i$ or $j \bmod 3$. Then $L_{i j}$ is on $Z_{k}$ and it meets $P_{k}$ and $P_{k+3}$ in two distinct points. It is therefore connected, by (3.2). Clearly it is on no connected plane on $E$ other than $P_{i}$ and $P_{j}$. The 12 lines $L_{i j}, j \neq i+3$, are the only connected lines on $E$, for by (3.4) any connected line on $E$ is on two distinct connected planes on $E$.

We write $\left\langle P_{i} \cup P_{j} \cup P_{k}\right\rangle=X_{i j k}$ for $1 \leqq i<j<k \leqq 6$. Then $X_{i j k}$ is a point of $M$, being identical with $\left\langle L_{i j} \cap P_{k}\right\rangle$. If two of the suffices $i, j$ and $k$ are congruent mod 3 then $X_{i j k}$ is one of the points $Z_{1}, Z_{2}$ and $Z_{3}$. The remaining eight points $X_{i j k}$ are all distinct, for on any one of them there can be only three 


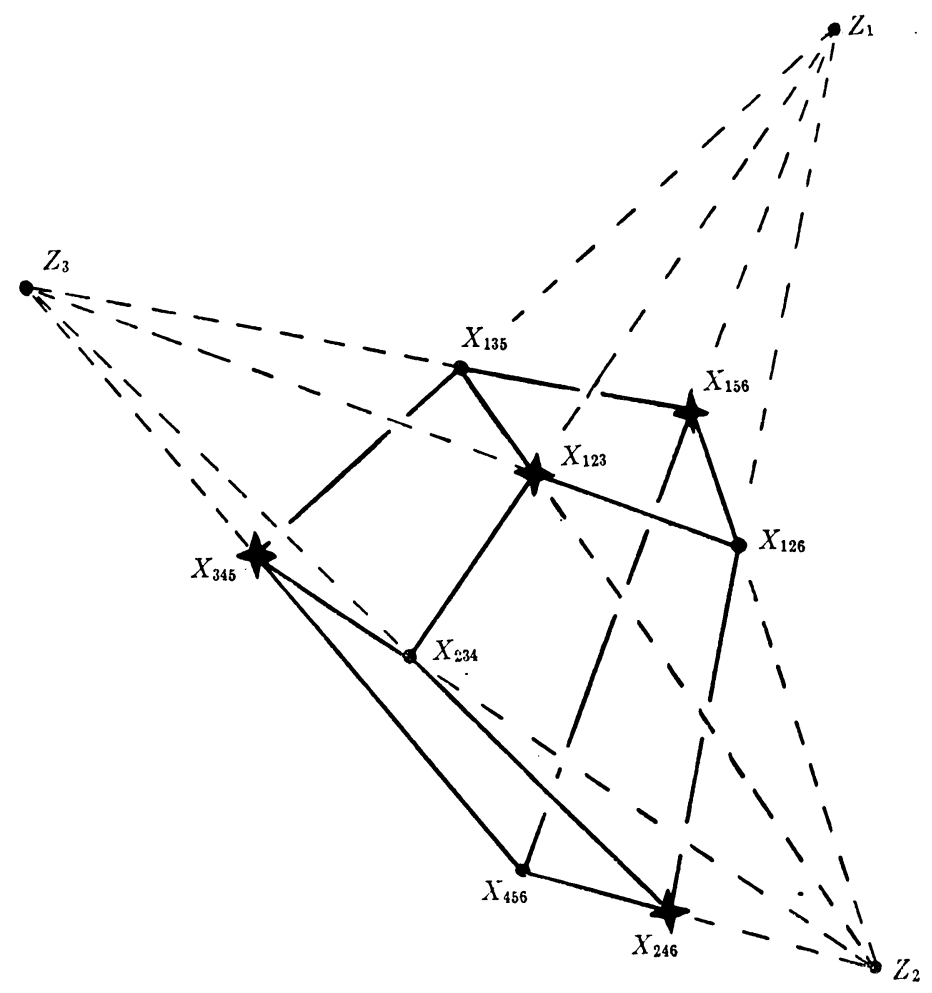

FIG. II

distinct planes such that each is on one of the lines $Z_{1} \cup Z_{2}, Z_{2} \cup Z_{3}$ and $Z_{3} \cup Z_{1}$. These eight points, together with $Z_{1}, Z_{2}$ and $Z_{3}$, are the only points on $E$. For any point on $E$ is on three distinct connected planes on $E$, by two applications of (3.4). (See Figure II.)

Consider the plane $P_{1}$. The only points on it are $Z_{2}, Z_{3}, X_{123}, X_{126}, X_{135}$ and $X_{156}$. We may adjust the notation so that $X_{123} \in C$. The other point of $C$ on $P_{1}$ can have no common connected line with $X_{123}$ and must therefore be $X_{156}$. We now find that $X_{246} \in C$ since this is the only point on $P_{2}$ having no common connected line with $X_{123}$. Proceeding in this way we find that $X_{i j k} \in C$ if and only if no two of the suffices are congruent mod 3 and the number of suffices less than 4 is odd. In Figures II, III and IV we represent points of $\boldsymbol{C}$ by four-pointed stars.

To construct a matroid having the structure just described we may use a method based on (2.3). We take $M$ to be a set of six cells in 1-1 correspondence with the planes $P_{i}$. Any point $X_{i j k}$ is represented by the set of those cells not corresponding to planes on $X_{i j k}$.

Suppose we have two paths $P Q R$ and $P R$ off $C$, where $Q$ is an elementary re-entrant path of the $k$ th kind with respect to $C$. Then we call the process 
of deriving one of the paths $P Q R$ and $P R$ from the other an elementary deformation of the $k$ th kind with respect to $C$. We say that two given paths $P^{\prime}$ and $P^{\prime \prime}$ off $\boldsymbol{C}$ are homotopic with respect to $\boldsymbol{C}$ (written $P^{\prime} \sim P^{\prime \prime}(\mathrm{C})$ ) if they are identical or if one can be derived from the other by a finite sequence of elementary deformations with respect to $\boldsymbol{C}$. Homotopy with respect to $\boldsymbol{C}$ is an equivalence relation. A path $P$ homotopic to a degenerate path with respect to $C$ is said to be null-homotopic with respect to $C$ (written $P \sim 0(\boldsymbol{C})$ ).

The null subset of $\boldsymbol{M}$ is clearly convex. If $\boldsymbol{C}$ is null we have only elementary deformations of the first and second kind to consider, and homotopy with respect to $\boldsymbol{C}$ becomes identical with the homotopy defined in the Introduction.

If $P$ is any path of $M$ we write $P^{-1}$ for the path obtained by taking the terms of $P$ in reverse order.

(5.2) If $P$ is any path off $C$ then $P P^{-1} \sim 0(C)$.

Proof. If possible choose $P$ so that the theorem fails and $P$ has the least number $s$ of terms consistent with this. If $s>1$ we can write $P=Q R$, where $Q$ and $R$ have each fewer than $s$ terms. Since $R R^{-1}$ and $Q Q^{-1}$ can be converted into degenerate paths by elementary deformations we have $P P^{-1}=Q R R^{-1} Q^{-1}$ $\sim Q Q^{-1} \sim 0$ (C). If $s=1$ then $P P^{-1}$ is an elementary re-entrant path of the first kind, and so $P P^{-1} \sim 0$ (C). The theorem follows.

(5.3) If PUR and $P V R$ are paths off $\boldsymbol{C}$ such that $U V^{-1} \sim 0$ (C), then $P U R \sim P V R(\mathbf{C})$.

Proof. By (5.2) we have $V^{-1} V=V^{-1}\left(V^{-1}\right)^{-1} \sim 0$ (C). Hence PUR $\sim P U V^{-1} V R \sim P V R(\mathrm{C})$.

(5.4) Let $\boldsymbol{C}$ be any convex subclass of a matroid $\boldsymbol{M}$. Let $S$ be a $d$-flat of $\boldsymbol{M}$ on a $(d+1)$-flat $T$ of $M$. Suppose all the points on $S$ and at least one other point on $T$ are members of $\boldsymbol{C}$. Then all the points on $T$ are members of $\boldsymbol{C}$.

Proof. Suppose the theorem false. Then we can find points $X \in C$ and $Y \notin C$, both on $T$ but not on $S$. The flat $X \cup Y$ is connected since otherwise $S \subset S \cup X \subset T$, contrary to (2.2). By (5.1) there is a path from $X$ to $Y$ on $X \cup Y$ whose second term, $X^{\prime}$ say, is not a member of $C$. By (2.5) the line $X \cup X^{\prime}$ has a point $X^{\prime \prime}$ in common with $S$. But $X^{\prime \prime} \in C$, by the definition of a convex subclass. This is contrary to hypothesis.

\section{Proof of the main theorem.}

(6.1) Let $\mathbf{C}$ be any convex subclass of a matroid $\boldsymbol{M}$ and let $P$ be any re-entrant path of $\boldsymbol{M}$ off $\boldsymbol{C}$. Then $P \sim 0(\boldsymbol{C})$.

Proof. Assume the theorem false. Let $P$ be any re-entrant path off $\mathbf{C}$ which is not null-homotopic with respect to $C$, and for which $d F(P)$ has the least value, $n$ say, consistent with this condition. For an arbitrary path $Q$ of $\boldsymbol{M}$ we call $d F(Q)$ the dimension of $Q$.

By far the most difficult part of the proof is that covered by the following lemma.

Lemma. Suppose $n \geqq 3$. Let $Q=(W, X, Y, Z, W)$ be a path off $\mathbf{C}$ of dimension 
n such that $W \cup X \cup Y$ and $Y \cup Z \cup W$ are connected planes and $W \cup Y$ is a disconnected line. Then $Q \sim 0(\mathrm{C})$.

Proof. Write $F_{1}=W \cup X \cup Y$ and $F_{2}=Y \cup Z \cup W$.

We note that if $Q^{\prime}=\left(W, X^{\prime}, Y, Z^{\prime}, W\right)$ is a path off $\boldsymbol{C}$ such that $X^{\prime}$ is on $F_{1}$ and $Y^{\prime}$ on $F_{2}$, then

$$
Q^{\prime} \sim Q(\mathbf{C})
$$

For

$$
Q^{\prime} \sim\left(W, X^{\prime}, Y\right)(Y, X, W)(W, X, Y)(Y, Z, W)(W, Z, Y)\left(Y, Z^{\prime}, W\right)(C)
$$

by (5.2). But $\left(W, X^{\prime}, Y\right)(Y, X, W)$ and $(W, Z, Y)\left(Y, Z^{\prime}, W\right)$ are re-entrant paths off $\boldsymbol{C}$ of dimension $<n$ and are therefore null-homotopic with respect to $\mathbf{C}$. Hence $Q^{\prime} \sim(W, X, Y)(Y, Z, W)=Q(\mathbf{C})$.

A transversal of dimension $n-1$ is a connected $(n-1)$-flat of $M$ which is on $F(Q)$ but not on both $W$ and $Y$. By (2.2) and (2.5) such a transversal meets each of $F_{1}$ and $F_{2}$ in a line. These two lines are connected, by (4.4).

A transversal of dimension $n-2$ is a connected $(n-2)$-flat of $M$ which is on $F(Q)$ but not on $W$ or $Y$. By (2.2) and (2.5) the transversal has just one point in common with each of $F_{1}$ and $F_{2}$. We call these two points the poles of the transversal.

Let $B$ be any transversal of dimension $n-2$, with poles $X^{\prime}$ on $F_{1}$ and $Z^{\prime}$ on $F_{2}$. Then $B$ is on two distinct connected $(n-1)$-flats of $M$ on $F(Q)$, by (3.4). Using (2.5) we find that each of these is on one, but not both, of $W$ and $Y$. Hence, by (2.2) they are $B \cup W$ and $B \cup Y$. They are transversals of dimension $n-1$. The flats $X^{\prime} \cup W, X^{\prime} \cup Y, Z^{\prime} \cup W$ and $Z^{\prime} \cup Y$ are their connected lines of intersection with $F_{1}$ and $F_{2}$. We note that a path $\left(W, X^{\prime}, Y\right.$, $\left.Z^{\prime}, W\right)$ exists.

Assume that $Q$ is not null-homotopic with respect to $C$.

Suppose $B$ is a transversal of dimension $n-2$ with poles $X^{\prime}$ on $F_{1}$ and $Z^{\prime}$ on $F_{2}$. Suppose further that neither $X^{\prime}$ nor $Z^{\prime}$ belongs to $C$. Then, by (5.1) there is a path $R$ off $\boldsymbol{C}$ from $X^{\prime}$ to $Z^{\prime}$ on $B$. Now $\left(W, X^{\prime}\right) R\left(Z^{\prime}, W\right)$ and $\left(X^{\prime}, Y, Z^{\prime}\right) R^{-1}$ are paths on the $(n-1)$-flats $B \cup W$ and $B \cup Y$ respectively. Hence their dimensions are less than $n$ and so they are null-homotopic with respect to $C$. Using (6.1a), (5.3) and (5.2) we find $Q \sim\left(W, X^{\prime}, Y, Z^{\prime}, W\right)$ $=\left(W, X^{\prime}\right)\left(X^{\prime}, Y, Z^{\prime}\right)\left(Z^{\prime}, W\right) \sim\left(W, Z^{\prime}\right) R^{-1} R\left(Z^{\prime}, W\right) \sim 0(\mathrm{C})$. This is contrary to assumption. We deduce that each transversal of dimension $n-2$ has at least one pole in $C$.

By (3.5) there is a transversal $A$ of dimension $n-1$ which is not on $Y$. Let its lines of intersection with $F_{1}$ and $F_{2}$ be $L_{1}$ and $L_{2}$ respectively. They are connected lines on $W$. By (3.2) there is a point $X^{\prime}$ of $\boldsymbol{M}-\boldsymbol{C}$ other than $W$ on $L_{1}$. By (3.5) there is a connected $(n-2)$-flat $B$ of $\boldsymbol{M}$ which is on $A$ and $X^{\prime}$ but not on $W$. Now $B$ is a transversal of dimension $n-2$. Let its pole on $L_{2}$ be $U_{2}$. Then $U_{2} \in C$. Similarly there is a transversal $B^{\prime}$ of dimension $n-2$ on $A$ having a point $Z^{\prime}$ of $\boldsymbol{M}-\boldsymbol{C}$ as its pole on $L_{2}$ and a point $U_{1}$ of $\boldsymbol{C}$ 


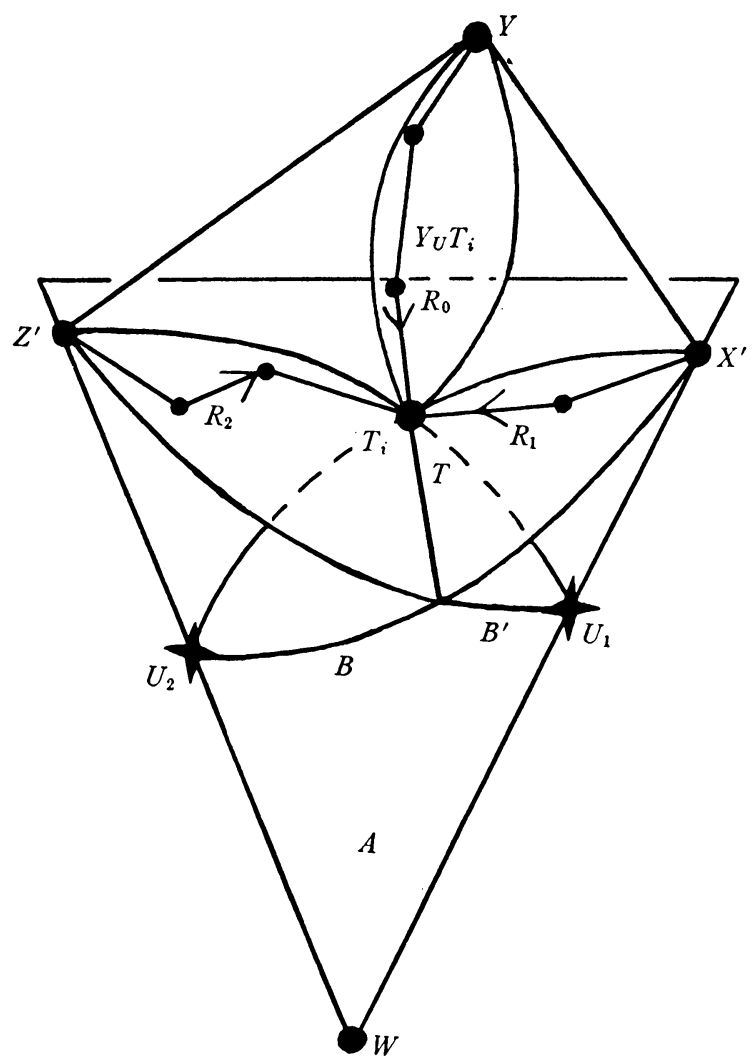

FIG. III

as its pole on $L_{1}$. (See Figure III.) We write $T=\left\langle B \cap B^{\prime}\right\rangle$. By (2.2) and (2.5) $T$ is an $(n-3)$-flat of $M$.

Let $S$ be the class of all members of $M-C$ on $T$. Since $T \subset B, X^{\prime} \in M-C$ and $U_{2} \in C$ it follows by (5.4) that $S$ is non-null.

Let $T_{i}$ be any point of $S$. Suppose that the flat $Y \cup T_{i}$ is connected. Then there is a path $R_{0}$ from $Y$ to $T_{i}$ on $Y \cup T_{i}$ which is off $C$, by (5.1). Similarly there is a path $R_{1}$ from $X^{\prime}$ to $T_{i}$ on $B$ and a path $R_{2}$ from $Z^{\prime}$ to $T_{i}$ on $B^{\prime}$, both $R_{1}$ and $R_{2}$ being off $C$. Now $\left(X^{\prime}, Y\right) R_{0} R_{1}^{-1}$ is a re-entrant path on the transversal $B \cup Y$ of dimension $n-1$, and $\left(Y, Z^{\prime}\right) R_{2} R_{0}^{-1}$ is a re-entrant path on the transversal $B^{\prime} \cup Y$ of dimension $n-1$. Hence both these paths are nullhomotopic with respect to $C$. Applying (6.1a), (5.3) and (5.2) we find $Q \sim\left(W, X^{\prime}, Y, Z^{\prime}, W\right)=\left(W, X^{\prime}\right)\left(X^{\prime}, Y\right)\left(Y, Z^{\prime}\right)\left(Z^{\prime}, W\right) \sim\left(W, X^{\prime}\right) R_{1} R_{0}^{-1} R_{0} R_{2}^{-1}$ - $\left(Z^{\prime}, W\right) \sim\left(W, X^{\prime}\right) R_{1} R_{2}^{-1}\left(Z^{\prime}, W\right)(C)$. But the last path is on the $(n-1)$-flat $A$ and is therefore null-homotopic with respect to $C$. Hence $Q \sim 0$ (C), contrary to assumption. We deduce that $Y \cup T_{i}$ has a separation $\left\{Y, T_{i}\right\}$. That is $Y \cup T_{i}$ is a disconnected line, and $Y$ and $T_{i}$ are the two points on it.

We can repeat the above argument with $B \cup Y$ replacing $A$. Instead of 
$B^{\prime}$ we then obtain a transversal $B^{\prime \prime}$ of dimension $n-2$ on $B \cup Y$ with its pole on $U_{2} \cup Y$ a member of $\boldsymbol{M}-\boldsymbol{C}$ and its pole on $X^{\prime} \cup Y$ a member of $\boldsymbol{C}$. We denote the $(n-3)$-flat $\left\langle B \cap B^{\prime \prime}\right\rangle$ by $T^{\prime}$. We find that any point $T_{j}^{\prime}$ of $M-C$ on $T^{\prime}$ is such that $W \cup T_{j}^{\prime}$ is a disconnected line. But, by $(2.5), B^{\prime \prime}$ has a point in common with the disconnected line $Y \cup T_{i}$, and this point can only be $T_{i}$. Hence $T_{i}$ is one of the points of $M-C$ on $T^{\prime}$.

We conclude that any point $T_{i}$ of $S$ is such that $W \cup T_{i}$ and $Y \cup T_{i}$ are disconnected lines.

Let $E$ be a connected flat of $M$ on $F_{1}$ and $F(Q)$ which is on some point of $S$ and has the least dimension consistent with this property. It is clear that either $F(Q)$ or one of its subsets satisfies these conditions. We have

$$
\begin{gathered}
n=d F(Q) \geqq d E \geqq 3, \\
d\langle E \cap T\rangle \geqq d E-3,
\end{gathered}
$$

by (2.2) and (2.5). Choose a point $N$ on $\langle E \cap T\rangle$, taking $N \in C$ if this is possible. By (3.5) there is a connected $(d E-1)$-flat $E^{\prime}$ of $M$ on $F_{1}$ and $E$ but not on $N$. By (2.2) and (2.5) $\left\langle E^{\prime} \cap T\right\rangle$ is a $(d\langle E \cap T\rangle-1)$-flat on $\langle E \cap T\rangle$. All the points of $M$ which are subsets of $\left\langle E^{\prime} \cap T\right\rangle$ belong to $C$, by the definition of $E$. By the choice of $N$ this implies that either $d\left\langle E^{\prime} \cap T\right\rangle=-1$ or $N \in C$. But in the latter case all the points on $\langle E \cap T\rangle$ belong to $C$, by (5.4), contrary to the definition of $E$. Hence $d\left\langle E^{\prime} \cap T\right\rangle=-1$ and therefore $d\langle E \cap T\rangle=0$. Hence $d E=3$, by $(6.1 \mathrm{~b})$ and (6.1c). Henceforth we use the symbol $T_{i}$ to denote the single point $\langle E \cap T\rangle$, which must be in $S$.

Suppose $n \geqq 4$. Then $F_{2}$ is not on $E$. By (3.5) there is a connected $(n-1)$ flat $E^{\prime \prime}$ of $\boldsymbol{M}$ on $F_{2}$ and $F(Q)$ but not on $T_{i}$. Write $F_{3}=\left\langle E^{\prime \prime} \cap E\right\rangle$. Then $F_{3}$ is a plane on $E$ and $W \cup Y$, by (2.2) and (2.5). By (3.5) there is a connected line $L$ on $E$ and $T_{i}$ having no common point with $W \cup Y$. Let its common points with $F_{1}$ and $F_{3}$ be $X_{1}$ and $X_{3}$ respectively. Neither of these is $T_{i}$. We note that $F_{3}=W \cup Y \cup X_{3}$, by (2.2). Now $X_{1} \cup W$ and $X_{1} \cup Y$ are connected lines by (4.3). Hence $X_{1} \cap W$ and $X_{1} \cap Y$ are both non-null, by (3.1). But we have shown that $W \cup T_{i}$ and $Y \cup T_{i}$ are disconnected lines. Hence $T_{i} \cap W$ and $T_{i} \cap Y$ are both null. But $L=X_{1} \cup T_{i}=X_{3} \cup T_{i}$, by (3.1). Hence $X_{3} \cap W$ and $X_{3} \cap Y$ are both non-null and therefore $F_{3}$ is connected.

By (5.1) there is a path $R$ from $Y$ to $W$ on $F_{3}$ which is off $C$. The re-entrant paths $(W, X, Y) R$ and $(Y, Z, W) R^{-1}$ are on $E$ and $E^{\prime \prime}$ respectively and so have dimensions $<n$. Hence they are null-homotopic with respect to $C$. Using (5.3) and (5.2) we find $Q=(W, X, Y)(Y, Z, W) \sim R^{-1} R \sim 0$ (C), contrary to assumption.

We deduce, using $(6.1 \mathrm{~b})$, that $n=3$. This implies $d T=0$. Hence $T$ is a point of $\boldsymbol{M}$, identical with $T_{i}$ and therefore a member of $\boldsymbol{M}-\boldsymbol{C}$. The three flats $W \cup Y, Y \cup T$ and $T \cup W$ are disconnected lines. The flat $W \cup Y \cup T$ is not a connected plane of $M$ by (4.3).

Any plane on $F(Q)$ has a point in common with each of the disconnected 
lines $W \cup Y, Y \cup T$ and $T \cup W$. It is therefore on one of these lines. Each line of $F(Q)$ is on a plane of $F(Q)$, by (2.2) and (2.3). It follows that each line on $F(Q)$ is on one of the points $W, Y$ and $T$.

Let $F$ be any transversal of dimension 2 . It meets $F_{1}$ and $F_{2}$ in connected lines $L_{1}$ and $L_{2}$ respectively. Let the points on $L_{1}$ other than $W$ or $Y$ be $X_{1}, \cdots, X_{k}$. By (3.4) there is a transversal $B_{i}$ of dimension 1 on $F$ and $X_{\imath}$ for each $i$. The line $B_{i}$ must be on $T$. Hence $B_{i}=X_{i} \cup T$, and $B_{i}$ is uniquely determined for each $i$. Let $X_{i}^{\prime}$ denote the point of intersection of $B_{i}$ and $L_{2}$. Since $B_{i}=T \cup X_{i}^{\prime}$ for each $i$ the $k$ points $X_{1}^{\prime}, \cdots, X_{k}^{\prime}$ are all distinct. But at most one point on each of the lines $L_{1}$ and $L_{2}$ belongs to $C$, and no transversal of dimension 1 has both its poles in $\boldsymbol{M}-\boldsymbol{C}$. Applying (3.2) we deduce that $k=2$. Moreover we can adjust the notation so that $\left(X_{1}, X_{2}^{\prime}\right) \in C$ and $\left(X_{2}, X_{1}^{\prime}\right) \in M-C$.

Distinct lines on $T$ and $F$ meet $L_{1}$ in distinct points, by (3.1). Hence the only connected lines on $T$ and $F$ are $B_{1}$ and $B_{2}$. But each point of $F$ is on two connected lines on $F$, and one of these is on $T$. Hence $X_{1}$ and $X_{2}^{\prime}$ are the only points of $\boldsymbol{C}$ on $F$. We thus prove that each connected plane on $F(Q)$ not on $W \cup Y$ is on just two points of $\boldsymbol{C}$.

Any connected line on $F_{1}$ is on a transversal of dimension 2, by (3.4). Hence it is on just three points, one of which is in $C$. Distinct lines on $F_{1}$ and $W$ (or $Y$ ) meet a given connected line on $F_{1}$ and $Y$ (or $W$ ) in distinct points, by (3.1). It follows that on $F_{1}$ there are just two connected lines on each of the points $W$ and $Y$. As each point on $F_{1}$ is on two connected lines on $F_{1}$ we deduce that $F_{1}$ is on just two points of $C$. Analogous results hold for $F_{2}$. Two distinct transversals of dimension 2 are both on $T$ and therefore meet $F_{1}$ in distinct lines, by (2.2). Accordingly there are just two connected planes on $F(Q)$ and $T \cup W$, and just two on $F(Q)$ and $Y \cup T$ (since $W \cup Y \cup T$ is not a connected plane).

It follows from these results that either $Q$ is an elementary re-entrant path of the fourth kind with respect to $C$ or there is a third connected plane $F_{3}$ on $F(Q)$ and $W \cup Y$. The first alternative must be rejected since it implies $Q \sim 0($ C) .

Let the points of intersection with $F_{3}$ of $B_{1}$ and $B_{2}$ be $X_{1}^{\prime \prime}$ and $X_{2}^{\prime \prime}$ respectively. These are both in $\boldsymbol{M}-\boldsymbol{C}$. Each is on two connected lines on $F_{3}$, one on $W$ and the other on $Y$, by (4.3). We have $\left(W, X, Y, X_{1}^{\prime \prime}, W\right)=(W, X$, $Y) \cdot\left(Y, X_{1}^{\prime \prime}, W\right) \sim 0(\boldsymbol{C})$. For otherwise we can repeat the first parts of the preceding proof with $\left(W, X, Y, X_{1}^{\prime \prime}, W\right)$ replacing $Q$ and obtain a contradiction, for the transversal $B_{2}$ then has both poles in $\boldsymbol{M}-\boldsymbol{C}$. Similarly $(Y, Z, W$, $\left.X_{1}^{\prime \prime}, Y\right)=(Y, Z, W)\left(W, X_{1}^{\prime \prime}, Y\right) \sim 0(\mathbf{C})$. Applying (5.3) and (5.2) we find $Q=(W, X, Y)(Y, Z, W) \sim\left(W, X_{1}^{\prime \prime}, Y\right)\left(Y, X_{1}^{\prime \prime}, W\right) \sim 0(\mathbf{C})$, contrary to assumption. The lemma follows.

We return to the path $P$ defined at the beginning of this proof. We note that $n=d F(P) \geqq 1$ since otherwise $P$ would be trivially null-homotopic with respect to $C$. We choose a connected $(n-1)$-flat $E$ of $\boldsymbol{M}$ which is on $F(P)$ 
and the origin $X_{0}$ of $P$. This choice is possible, by (3.3).

Let $R=\left(X_{0}, \cdots, X_{m}, X_{0}\right)$ be any re-entrant path with the same origin as $P$ on $F(P)$. We write $u(R)$ for the number of terms of $R$, counting repetitions, which are not on $E$. If $u(R)>0$ we write $X_{i}$ for the first term of $R$ which is not on $E$. We then write $v(R)=d\left(X_{i-1} \cup X_{i} \cup X_{i+1}\right)$, taking $X_{m+1}$ $=X_{0}$ if $i=m$. If $u(R)=0$ we write $v(R)=0$.

Henceforth we suppose $R$ chosen so as to satisfy the following conditions:

(i) $R \sim P(\mathrm{C})$,

(ii) $u(R)$ has the least value consistent with (i),

(iii) $v(R)$ has the least value consistent with (i) and (ii).

We consider first the case $u(R)>0$. Then $v(R)>0$. We may conveniently write $R$ in the form $R_{1}\left(X_{i-1}, X_{i}, X_{i+1}\right) R_{2}$, noting that $R_{1}$ is a path on.. IVe write also $F=X_{i-1} \cup X_{i} \cup X_{i+1}$.

Suppose $v(R)=1$. Then $F$ is a connected line. If $X_{i+1}=X_{i-1}$ we have $R \sim R_{1} R_{2}(C)$, by an elementary deformation of the first kind. This is impossible since $u\left(R_{1} R_{2}\right)<u(R)$. If $X_{i+1} \neq X_{i-1}$ then $\left(X_{i-1}, X_{i}, X_{i+1}, X_{i-1}\right)$ is an elementary re-entrant path of the second kind with respect to $C$. Applying (5.3) we find $R \sim R_{1}\left(X_{i-1}, X_{i+1}\right) R_{2}(\mathrm{C})$. This is impossible since

$$
u\left(R_{1}\left(X_{i-1}, X_{i+1}\right) R_{2}\right)<u(R) .
$$

Suppose $v(R)=2$. Then $F$ is a connected plane on $F(Q)$. It meets $E$ in a line $L$, by (2.2) and (2.5). Let $Z$ be the point of intersection of the lines $L$ and $X_{i} \cup X_{i+1}$ on $F$. We discuss first the case $Z \in M-C$. In this case we define $Q$ as the degenerate path $(Z)$ if $Z=X_{i+1}$ and as the path $\left(Z, X_{i+1}\right)$ otherwise. Then $\left(X_{i}, X_{i+1}\right) Q^{-1}\left(Z, X_{i}\right)$ is an elementary re-entrant path of the first or second kind. Hence $R \sim R_{1}\left(X_{i-1}, X_{i}, Z\right) Q R_{2}(C)$, by (5.3). If $L$ is connected we have $\left(X_{i-1}, X_{i}, Z, X_{i-1}\right) \sim 0(\mathrm{C})$ and therefore $R \sim R_{1}\left(X_{i-1}, Z\right) Q R_{2}(\mathrm{C})$, by (5.3). If $L$ is not connected it is on a connected plane $F^{\prime}$ of $M$ on $E$, by (4.2). We can find a connected line $L^{\prime}$ on $X_{i-1}$ and $F^{\prime}$, and a point $T$ of $\boldsymbol{M}-\boldsymbol{C}$ distinct from $X_{i-1}$ on $L^{\prime}$. Then $T \cup X_{i-1}$ and $T \cup Z$ are connected lines, by (4.3). Using the lemma and the definition of $n$ we find $\left(X_{i-1}, X_{i}, Z, T, X_{i-1}\right)$ $\sim 0$ (C). Hence $R \sim R_{1}\left(X_{i-1}, T, Z\right) Q R_{2}(\mathrm{C})$, by (5.3). So whether $L$ is connected or not we have $R \sim R_{3} Q R_{2}(\mathrm{C})$, where $R_{3}$ is on $E$. This is impossible since $u\left(R_{3} Q R_{2}\right)<u(R)$.

We go on to the case $Z \in C$, illustrated in Figure IV. By (3.4) there is a connected line $L^{\prime}$ other than $X_{i} \cup X_{i+1}$ on $X_{i+1}$ and $F$. If $L^{\prime}$ is on $X_{i-1}$ we have $R \sim R_{1}\left(X_{i-1}, X_{i+1}\right) R_{2}(C)$, using (5.3) with the elementary re-entrant path $\left(X_{i-1}, X_{i}, X_{i+1}, X_{i-1}\right)$ of the second kind. This is impossible since $u\left(R_{1}\left(X_{i-1}, X_{i+1}\right) R_{2}\right)<u(R)$. Hence $L^{\prime}$ must meet the lines $X_{i-1} \cup X_{i}$ and $L$ in distinct points $U$ and $V$ respectively. Since $Z \in C$ and $X_{i-1} \in M-C$ we have $V \in \boldsymbol{M}-\boldsymbol{C}$.

Suppose $U \in M-C$. Using (5.3) with elementary re-entrant paths of the first and second kinds we find 
$R \sim R_{1}\left(X_{i-1}, U, X_{i}, X_{i+1}\right) R_{2} \sim R_{1}\left(X_{i-1}, V, U, X_{i+1}\right) R_{2} \sim R_{1}\left(X_{i-1}, V, X_{i+1}\right) R_{2}(\mathrm{C})$

This is impossible since $u\left(R_{1}\left(X_{i-1}, V, X_{i+1}\right) R\right)<u(R)$.

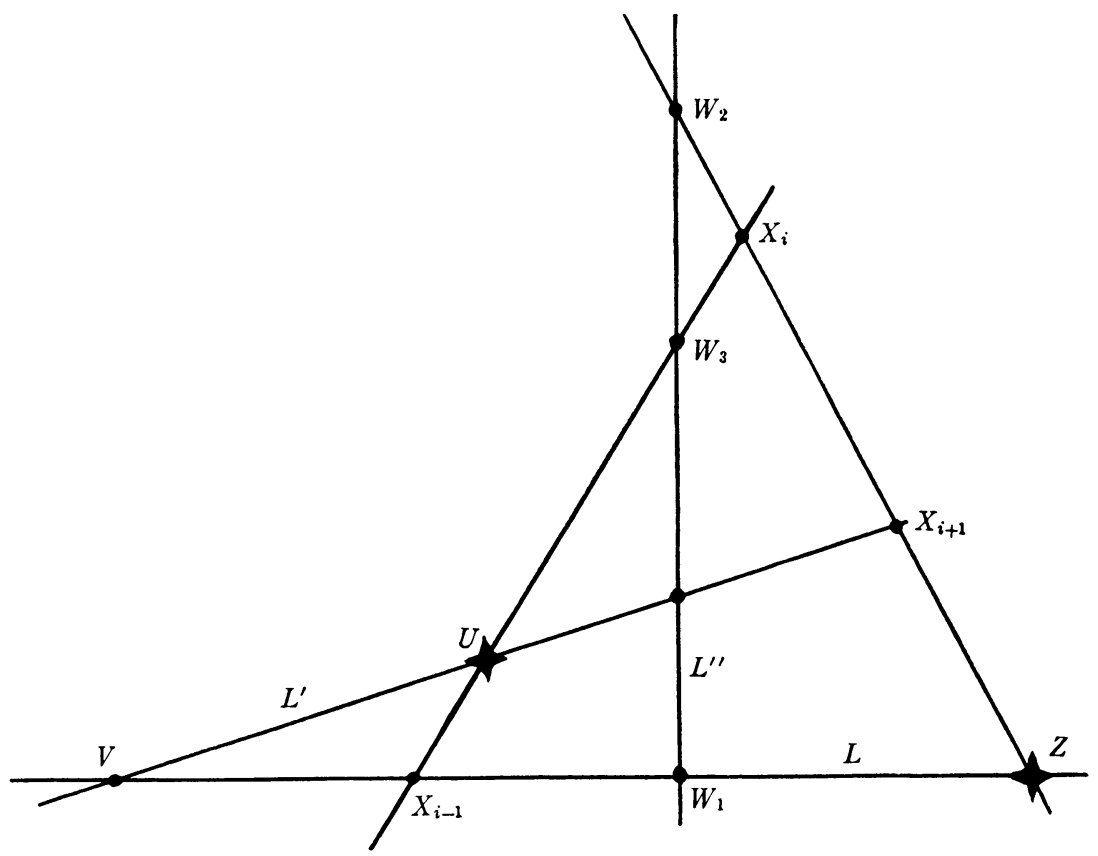

FIG. IV

Suppose $U \in C$. It may happen that each connected line on $F$ is on either $U$ or $Z$. Then $\left(X_{i-1}, X_{i}, X_{i+1}, V, X_{i-1}\right)$ is an elementary re-entrant path of the third kind with respect to $C$. Using (5.3) we have $R \sim R_{1}\left(X_{i-1}, V, X_{i+1}\right) R_{2}$ (C), which is impossible, as before. Hence there is a connected line $L^{\prime \prime}$ on $F$ which is not on $U$ or $Z$. If $L^{\prime \prime}$ is on $X_{i+1}$ we can substitute it for $L^{\prime}$ in the preceding argument and so reduce to the case $U \in M-C$. We may therefore suppose $L^{\prime \prime}$ is not on $X_{i+1}$.

If $L^{\prime \prime}$ is on $X_{i}$ it meets $L$ in a point $W_{1}$ distinct from $X_{i-1}$ and $Z$. Writing $R^{\prime}=R_{1}\left(X_{i-1}, W_{1}, X_{i}, X_{i+1}\right) R_{2}$ we have $R^{\prime} \sim R(\mathrm{C})$, by (5.3). If $L^{\prime \prime}$ is not on $X_{i}$ it meets $X_{i} \cup X_{i+1}$ in a point $W_{2}$ distinct from $X_{i}, X_{i+1}$ and $Z$. If $L^{\prime \prime}$ is then on $X_{i-1}$ we write $R^{\prime}=R_{1}\left(X_{i-1}, W_{2}, X_{i+1}\right) R_{2}$ and have

$$
R^{\prime} \sim R_{1}\left(X_{i-1}, W_{2}, X_{i}, X_{i+1}\right) R_{2} \sim R(C),
$$

by (5.3). If instead $L^{\prime \prime}$ is not on $X_{i-1}$ it meets the lines $L, X_{i} \cup X_{i+1}$ and $X_{i-1}$ $\cup X_{i}$ in distinct points $W_{1}, W_{2}$ and $W_{3}$ respectively of $\boldsymbol{M}-\boldsymbol{C}$. We then write $R^{\prime}=R_{1}\left(X_{i-1}, W_{1}, W_{2}, X_{i+1}\right) R_{2}$ and have $R^{\prime} \sim R_{1}\left(X_{i-1}, W_{1}, W_{3}, W_{2}, X_{i}, X_{i+1}\right) R_{2}$ $\sim R_{1}\left(X_{i-1}, W_{3}, X_{i}, X_{i+1}\right) R_{2} \sim R(C)$, by (5.3). For each of these three possibilities we have $R^{\prime} \sim R(C), u\left(R^{\prime}\right)=u(R)$ and $v\left(R^{\prime}\right)=v(R)=2$. Hence we may 
replace $R$ by $R^{\prime}$ in the preceding argument. This reduces the problem to the case $U \in M-C$, which we have found to lead to a contradiction.

We now consider the case $v(R)>2$. By (3.3) there is a connected plane $K$ on $X_{i-1} \cup X_{i}$ and the connected $v(R)$-flat $F$. This plane meets $E$ in a line $L$. Choose a point $T$ distinct from $X_{i-1}$ on $L$ and if possible in C. By (3.5) there is a connected $(v(R)-1)$-flat $F^{\prime}$ on $X_{i} \cup X_{i+1}$ and $F$ but not on $T$. Now $F^{\prime}$ is not on $X_{i-1}$, for otherwise we would have $F \subseteq F^{\prime}$, contrary to (2.2). Hence $F^{\prime}$ meets $L$ in a point $T^{\prime}$ distinct from $X_{i-1}$ and $T$. It follows that $L$ is connected, by (3.2), and that $T^{\prime} \in M-C$. The flats $K$ and $F^{\prime}$ intersect in a line $L^{\prime}$ on $X_{i}$ and $T^{\prime}$. If $L^{\prime}$ is connected we write

$$
R^{\prime}=R_{1}\left(X_{i-1}, T^{\prime}, X_{i}, X_{i+1}\right) R_{2}
$$

and have $R^{\prime} \sim R(C)$, by (5.3). If $L^{\prime}$ is not connected it is on a connected plane $K^{\prime}$ on $F^{\prime}$, by (4.2). $K^{\prime}$ meets $E$ in a connected line $L^{\prime \prime}$ on $T^{\prime}$, by (4.4). We can find a point $U$ on $L^{\prime \prime}$ distinct from $T^{\prime}$ and in $M-C$. The flat $U \cup X_{i}$ is a connected line, by (4.3). Using the lemma and the definition of $n$ we find $\left(T^{\prime}, U, X_{i}, X_{i-1}, T^{\prime}\right) \sim 0(\mathrm{C})$. In this case we write

$$
R^{\prime}=R_{1}\left(X_{i-1}, T^{\prime}, U, X_{i}, X_{i+1}\right) R_{2}
$$

Then by (5.2) and (5.3) we have

$$
\begin{aligned}
R^{\prime} \sim R_{1}\left(X_{i-1}, T^{\prime}, U, X_{i}, X_{i-1}, T^{\prime},\right. & \left.X_{i-1}, X_{i}, X_{i+1}\right) R_{2} \\
& \sim R_{1}\left(X_{i-1}, T^{\prime}, X_{i-1}, X_{i}, X_{i+1}\right) R_{2} \sim R(\mathrm{C}) .
\end{aligned}
$$

So whether $L^{\prime}$ is connected or not we have $R^{\prime} \sim R(\mathbf{C}), u\left(R^{\prime}\right)=u(R)$ and $v\left(R^{\prime}\right)$ $<v(R)$, which is contrary to the definition of $R$.

From the above analysis we deduce that $u(R)=0$. Hence $R$ is on $E$ and has dimension $<n$. Hence $P \sim R \sim 0(\boldsymbol{C})$, contrary to assumption. The theorem follows.

7. Special cases. With $C$ null in (6.1) we find that every re-entrant path in a matroid $M$ is null-homotopic, as stated in the Introduction.

In applying this result to the circuit-matroid $M$ of a graph $G$ we must remember that a path in $\boldsymbol{M}$ corresponds to a sequence of circuits of $G$ such that any two consecutive circuits form a nonseparable subgraph of rank 2 . It can be shown that such a subgraph is made up of three arcs such that any two have both ends but no other edge or vertex in common. Each of the elementary deformations by which a re-entrant sequence of circuits can be transformed into a sequence with only one member operates within some nonseparable subgraph of rank $\leq 3$.

\section{REFERENCE}

1. Hassler Whitney, The abstract properties of linear dependence, Amer. J. Math. vol. 57 (1935) pp. 507-533.

University OF TORONTO, TORONTO, ONT. 